Research Paper

\title{
Depletion of WNT10A Prevents Tumor Growth by Suppressing Microvessels and Collagen Expression
}

\author{
Motona Kumagai ${ }^{1}$, Xin Guo ${ }^{1}$, Ke-Yong Wang2 ${ }^{2}$, Hiroto Izumi ${ }^{3}$, Manabu Tsukamoto ${ }^{4}$, Tamiji Nakashima ${ }^{5}$, \\ Takashi Tasaki², Nozomu Kuroseㄹ, Hidetaka Uramoto6, Yasuyuki Sasaguri7, Kimitoshi Kohno9, Sohsuke \\ Yamada ${ }^{1 凶}$ \\ 1. Department of Pathology and Laboratory Medicine, Kanazawa Medical University, Ishikawa 920-0293, Japan \\ 2. Shared-Use Research Center, School of Medicine, University of Occupational and Environmental Health, Kitakyushu 807-8555, Japan \\ 3. Department of Occupational Pneumology, School of Medicine, University of Occupational and Environmental Health \\ 4. Department of Orthopaedic Surgery, School of Medicine, University of Occupational and Environmental Health \\ 5. Department of Human, Information and Life Sciences, School of Medicine, University of Occupational and Environmental Health \\ 6. Department of Thoracic Surgery, Kanazawa Medical University, Ishikawa 920-0293, Japan \\ 7. Department of Pathology and Cell Biology, School of Medicine, University of Occupational and Environmental Health \\ 8. Laboratory of Pathology, Fukuoka Tokushukai Hospital, Fukuoka 816-0864, Japan \\ 9. Asahi-Matsumoto Hospital, Kitakyushu 800-0242, Japan.
}

$\triangle$ Corresponding authors: Sohsuke Yamada, M.D., Ph.D., Department of Pathology and Laboratory Medicine, Kanazawa Medical University, 1-1 Uchinada, Ishikawa, 920-0293, Japan. Tel: 81-76-218-8264; Fax: 81-76-286-1207; and E-mail: sohsuke@kanazawa-med.ac.jp; \& Xin Guo, M.D., Ph.D., Department of Pathology and Laboratory Medicine, Kanazawa Medical University, 1-1 Uchinada, Ishikawa, 920-0293, Japan. Tel: 81-76-218-8021; Fax: 81-76-286-1207; and E-mail: tianqi11211216@yahoo.co.jp.

(C) Ivyspring International Publisher. This is an open access article distributed under the terms of the Creative Commons Attribution (CC BY-NC) license (https://creativecommons.org/licenses/by-nc/4.0/). See http://ivyspring.com/terms for full terms and conditions.

Received: 2018.05.01; Accepted: 2018.09.06; Published: 2019.01.29

\begin{abstract}
Background: We recently reported that WNT10A plays a pivotal role in wound healing by regulating collagen expression/synthesis, as the depletion of WNT10A dramatically delays skin ulcer formation. WNT signaling also has a close correlation with the cancer microenvironment and proliferation, since tumors are actually considered to be 'unhealing' or 'overhealing' wounds. To ascertain the in vivo regulatory functions of WNT10A in tumor growth, we examined the net effects of WNT10A depletion using Wnt lOa-deficient mice (Wnt $\left.10 a^{--}\right)$.

Methods and Results: We subjected C57BL/6] wild-type (WT) or Wnt $10 a^{-1-}$ mice to murine melanoma B16-F10 cell transplantation. Wnt $10 a^{-1-}$ mice showed a significantly smaller volume of transplanted melanoma as well as fewer microvessels and less collagen expression and more necrosis than WT mice.

Conclusions: Taken together, our observations suggest that critical in vivo roles of Wnt lOa-depleted anti-stromagenesis prevent tumor growth, in contrast with true wound healing/scarring.
\end{abstract}

Key words: WNT10A, Wnt10a-deficient mice (Wnt10a-/-), tumor growth, microvessel, collagen expression

\section{Introduction}

WNT10A is a secreted glycoprotein and belongs to the highly conserved WNT glycoproteins family that includes 19 members identified so far in humans, which take part in various physiological and pathological processes through two major signaling pathways: the canonical $\beta$-catenin-dependent pathway and non-canonical $\beta$-catenin-dependent pathway [1-3]. This glycoprotein is a novel angio/stromagenic factor and has a key role in morpho/organogenesis and tumorigenesis by regulating fibrogenic factors [4-7]. Generally, WNT10A is primarily studied in the deregulation of ectodermal tissues relating to a variety of odonto-onychal dermal dysplasias [8-9]. Mutations in the WNT10A gene are closely associated with a broad range of ectodermal defects, such as tooth morphogenesis, hair follicle development, papillae of the tongue and sweat gland, nail formation, and regeneration of the epidermis [9-11].

Our recent results showed that the deficiency of WNT10A obviously postponed wound healing of the dorsal skin in mice, suggesting that WNT10A signaling can play a pivotal in vivo role in wound healing by regulating the expression and synthesis of collagen, as a fibrogenic factor [12]. There are remarkable cellular parallels between skin wounds 
and malignant tumors, which have been supported by various studies and extended to the cellular and molecular level [13]. Wound granulation consists of a large number of fibroblasts/myofibroblasts and newly formed blood vessels admixed with inflammatory cells, similar to the components of tumor stroma, which is very important for tumor progression. However, it is crucial that the non-self-limited processes of stromagenesis be activated in an exaggerated and prolonged manner in malignant tumors, promoting malignant cell proliferation, invasion and metastasis [13]. Therefore, tumors have always been seen as "unhealing wounds" or "overhealing wounds" [14].

The production of extracellular matrix (ECM) is known to be essential to the formation of granulation tissues in the repair process of wounds. The ECM, including several types of collagen, is mainly secreted by fibroblasts and/or myofibroblasts [15]. WNT10A expression was specifically observed in human keloid dermal myofibroblasts that immunohistochemically display the specific expression of a-smooth muscle actin (a-SMA) but not in normal skin dermal fibrocytes [16]. Thus, WNT10A signaling may be involved in the progression of malignant tumor via stromagenesis, including fibrogenesis and angiogenesis.

The in vivo roles of WNT10A in malignant tumor are poorly understood. In the present study using a murine melanoma transplantation model, we investigated the in vivo net effects of WNT10A in skin malignant tumor, with our findings suggesting that WNT10A may promote tumor growth through stimulating collagen expression to accelerate tumor stromagenesis.

\section{Materials and Methods}

\section{Animals and generation of Wnt $10 a-k n o c k o u t$ (Wnt 10a ${ }^{-l-}$ ) mice}

Wnt10a-targeted embryonic stem cells (ESCs) were obtained from the School of Veterinary Medicine, University of California, Davis (UC Davis, CA, USA). The knockout deleted 12663 bp (74791516 to 74804179 of mouse chromosome 1, genome build 38, Clone ID:14810A), which included the entire WNT10A coding region. The knockout construct replaced the Wnt10a gene in ES cells (C57BL/6 background) with a ZEN-Ub1 cassette that introduced a bacterial lacZ code at the natural Wnt10a translation initiation codon (in exon 1) and a downstream neomycin phosphotransferase gene (NEOr) driven by the human ubiquitin $\mathrm{C}$ gene promoter (hUBCpro). The neomycin resistance gene was bracketed by a locus of X-over P1 sequence from bacteriophage P1
(loxP) sequences for the convenient removal of the NEOr selection code. To generate chimeric mice, these ESC clones were microinjected into C57BL/6 blastocysts at NPO Biotechnology Research and Development, Osaka University (Osaka, Japan). Successful knockout was determined by reverse transcription polymerase chain reaction (RT-PCR) genotyping (http://www.velocigene.com/komp/ detail/14810). Heterozygous $W n t 10 a^{+/-}$females were then bred with $W n t 10 a^{+/-}$males, and $W n t 10 a^{-/-}$mice were born at the expected Mendelian ratio.

Experiments were performed in 10- to 12-week-old male $W n t 10 a^{-/-}$mice, and wild-type (WT) C57BL/6J mice (Charles River, Yokohama, Japan) were used to provide a control group in some experiments ( $\mathrm{n}=8$ to 12 mice per experiment). Animals were provided their diet and water ad libitum and maintained on a 12-h light/dark cycle. All animal experiments were conducted according to the Laboratory Animal Research Center at University of Occupational and Environmental Health School of Medicine. All surgery was performed under anesthetization using a mixture of ketamine $50 \mathrm{mg} / \mathrm{kg}$ (Daiichi Sankyo Co., Tokyo, Japan) and medetomidine $1 \mathrm{mg} / \mathrm{kg}$ (Meiji Yakuhin Co., Tokyo, Japan) as previously described [16-19].

The Ethics Committee of Animal Care and Experimentation, Kanazawa Medical University, approved the protocols. They were performed according to the Institutional Guidelines for Animal Experiments and the Law (no. 105) and Notification (no. 6) of the Japanese Government. The investigation conformed to the Guide for the Care and Use of Laboratory Animals published by the US National Institutes of Health (NIH Publication No. 85-23, revised 1996).

\section{Cell lines and culture conditions}

Mouse melanoma cell line B16-F10 cells were obtained from ATCC and cultured in Dulbecco's modified Eagle's minimal essential medium (DMEM). This medium was purchased from Nissui Seiyaku (Tokyo, Japan) and contained 10\% fetal bovine serum (FBS), $1 \%$ penicillin and $1 \%$ streptomycin. Cell lines were maintained in a $5 \% \mathrm{CO}_{2}$ atmosphere at $37^{\circ} \mathrm{C}$.

\section{Murine melanoma B 16-F 10 cells transplantation model}

Ten-week-old male mice were used for this model of tumor subcutaneous implanted. Mice were injected with $100 \mu \mathrm{l}\left(1 \times 10^{5}\right.$ cells $)$ of B16-F10 cells suspension at 2 separate dorsal sites. The tumor volume was measured using the two principal perpendicular diameters: $V=$ length $(\mathrm{mm}) \times$ [width $(\mathrm{mm})]^{2} \times 1 / 2$. At day 21 post-transplantation, mice 
were killed in a fed state by intraperitoneal anesthetization with an overdose of ketamine (100 $\mathrm{mg} / \mathrm{kg}$ ) (Daiichi Sankyo Co.) and medetomidine (2 $\mathrm{mg} / \mathrm{kg}$ ) (Meiji Yakuhin Co.). The tumors were then formalin-fixed and embedded in paraffin for a histologic examination [20-22].

\section{Histopathology and immunohistochemistry (IHC)}

The WT and Wnt10a-/- mice were killed in a fed state by intraperitoneal anesthetization with an overdose of ketamine $(100 \mathrm{mg} / \mathrm{kg}$ ) (Daiichi Sankyo Co.) and medetomidine $(2 \mathrm{mg} / \mathrm{kg}$ ) (Meiji Yakuhin Co.), and tumor tissues in the dorsal skin were removed, formalin-fixed in 10\% neutral buffered formalin for $24 \mathrm{~h}$, and embedded in paraffin for a histological examination. Tissue specimens were stained with hematoxylin and eosin (H\&E) or Masson's trichrome or prepared as immunohistochemistry (IHC) samples in sequential sections after fixation [23-25]. Frozen sections of healing skin were also produced for an immunofluorescence evaluation. Necrotic areas were defined as blurry, unstructured red dye regions fused by the dissolved necrotic cells and disintegrating stroma. The necrotic area/tumor area ratio was evaluated using an Olympus VS120 Virtual system (Olympus, Tokyo, Japan).

To determine the number of myofibroblasts and microvessels in wound healing lesions and tumor tissue, immunohistochemical staining was performed with monoclonal mouse anti-human a-SMA (dilution 1:150; Dako, Tokyo, Japan) for myofibroblasts and mouse monoclonal anti-CD31 antibody (dilution 1:500; Dako) for endothelial cells. The number of activated myofibroblasts and microvessels was

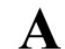

counted in 10 randomly selected fields of sections of healing skin and tumor lesions, respectively (original magnification: $x 400)$, as previously described [24-25]. To further assess the importance of WNT signaling in wound healing regulation, $\beta$-catenin and WNT10A immunohistochemical staining were performed using rabbit polyclonal anti- $\beta$-catenin antibody (dilution 1:500, AC $121^{\circ} \mathrm{C}$; Abcam, Tokyo, Japan) and rabbit polyclonal anti-WNT10A antibody (dilution 1:5000). We further performed IHC using the rabbit polyclonal anti-Type I collagen (dilution 1:500; Abcam) and rabbit polyclonal anti-Type III collagen (dilution 1:200; Proteintech, Rosemont, IL, USA).

\section{Statistical analyses}

Results are expressed as the means \pm standard error (SE). Significant differences were analyzed using Student's $t$-test, Welch's $t$-test or a one-way analysis of variance (ANOVA) where appropriate. In all cases in which an ANOVA was applied for non-parametric data, Tukey's multiple comparison post hoc test was used [16, 23-25]. Values of $P<0.05$ were considered statistically significant.

\section{Results}

\section{Deletion of WNT10A resulted in the suppression of tumor growth on the murine melanoma B 16-F10 cell transplantation model.}

In marked contrast to the murine wound healing model, Wnt10a-/- mice grossly revealed a significantly smaller volume of pigmented (black in color) tumor (WT $2870.6 \pm 690.5 \mathrm{~mm}^{3}$ vs. Wnt10a-/- $690.5 \pm 190.5$ $\mathrm{mm}^{3} ; P<0.001$ ) (Figure 1) than WT mice in the murine melanoma B16-F10 cell transplantation model.
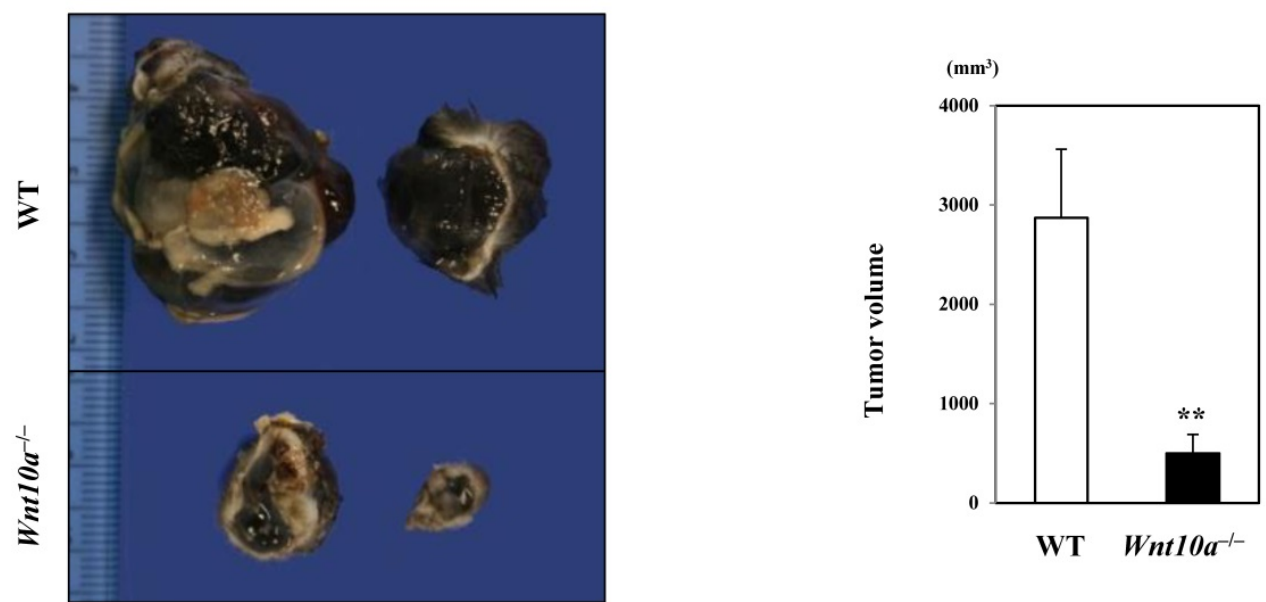

Figure 1. Wnt 10a-l- mice showing a significantly smaller volume of transplanted melanoma than WT mice. (A) The cut surface. (B) The transplanted Wnt $10 a^{-1-}$ mice showed a significantly smaller volume of pigmented (black in color) tumor than WT mice, which was confirmed by quantitative analyses ( $\mathrm{n}=8$ mice per group). Values are means \pm SE. $* * P<0.001$. 
Conversely, the transplanted melanomas in $W n t 10 a^{-/-}$mice contained significantly larger necrotic areas (green line in HE) than did those in WT mice (WT $26.2 \% \pm 7.2 \%$ vs. Wnt $10 a^{-/-} 43.2 \% \pm 3.7 \%$; $P<$ 0.05 ) (Figure 2).

\section{Deletion of WNT10A suppressed tumor stromagenesis.}

Microscopic findings showed that the pigmented melanoma in Wnt10a-/- mice was significantly associated with repressed proliferation of a-SMA- or CD31-positive microvessels (Figure 3) compared to WT mice, although only in cases where WNT10A and $\beta$-catenin co-expression was present, especially in the tumor vessels (Figure 3). Accordingly, there were significantly fewer microvessels in Wnt10a-/- mice than in WT mice (WT $5.4 \pm 0.8$ per $0.01 \mathrm{~mm}^{2}$ vs. Wnt10a-/- $2.0 \pm 0.5$ per $0.01 \mathrm{~mm}^{2} ; P<0.0001$ ) (Figure $3)$.

\section{Fibrosis and collagen expressions were reduced in Wnt $10 a^{-1-}$ mice}

Masson's trichrome staining revealed significantly smaller amounts of blue-stained collagenous stroma (i.e. more suppressed fibrosis/fibrogenesis) in the transplanted tumor lesions of Wnt10a-/- mice than in those of WT mice (Figure 4). Accordingly, IHC showed that Wnt10a-/melanomas had significantly lower expression of Type I/III collagen than WT ones, a finding that was observed in a large number of tumor vessel walls (Figure 4).

A

H\&E

$\mathbf{H \& E}$

B
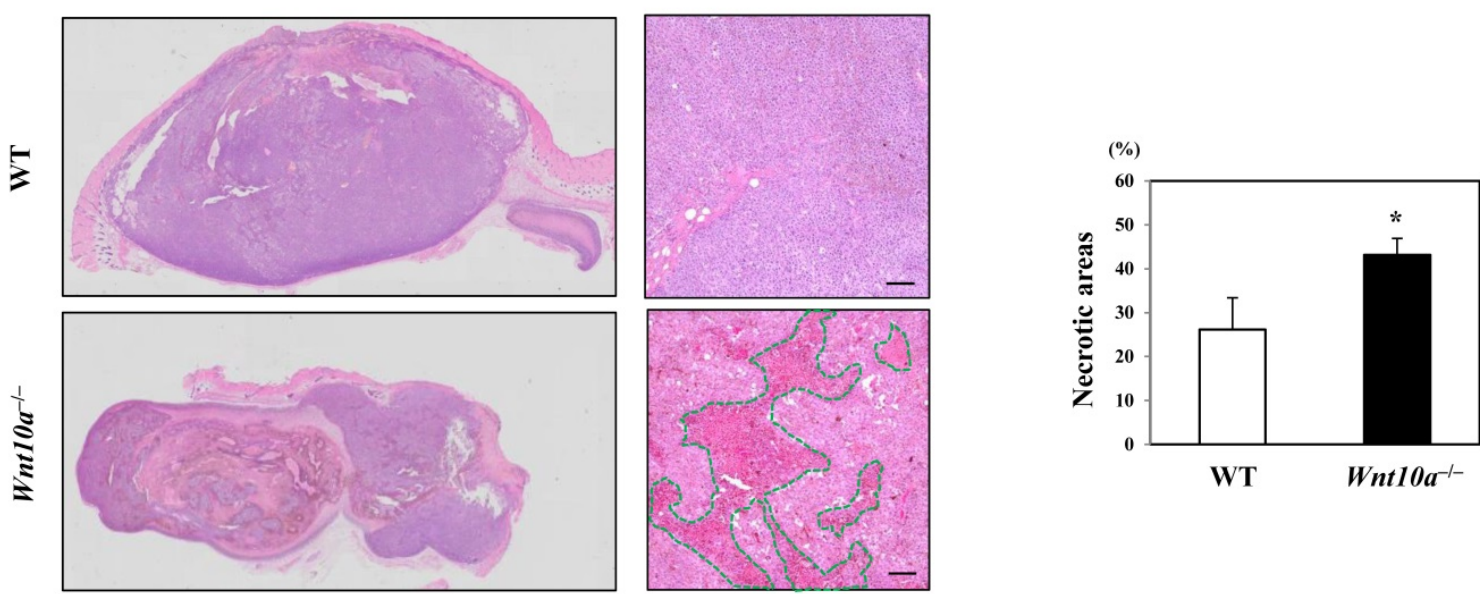

Figure 2. Wnt 10a-l- mice showed increased necrosis in the murine melanoma B16-F10 cell transplantation model. (A) Representative photomicrographs of H\&E-stained sections in the murine melanoma B16-F10 cell transplantation model, bar $=100 \mu \mathrm{m}$. (B) The transplanted melanomas in Wnt $/ 0 a^{-1-}$ mice contain significantly larger necrotic areas (green line in $\mathrm{HE}$ ) than those in WT mice.

A

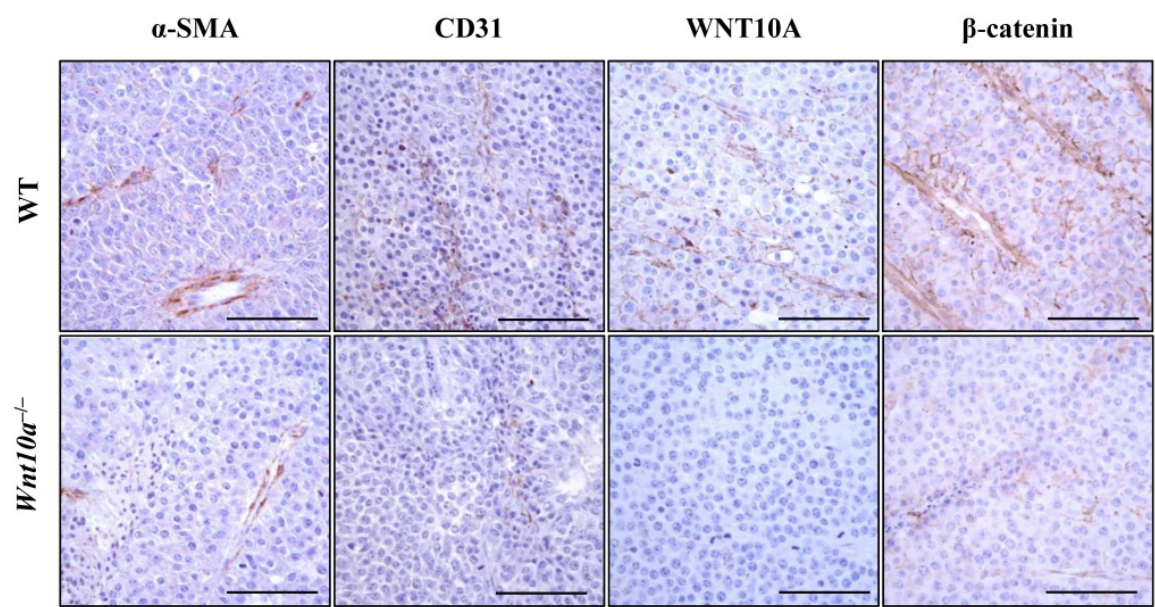

B

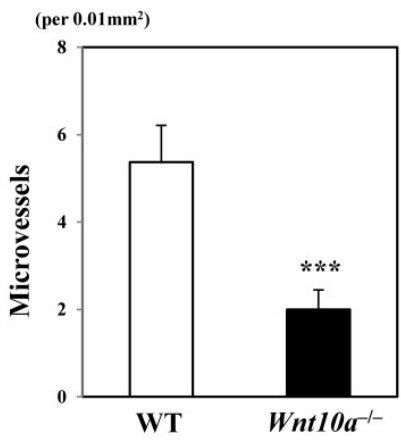

Figure 3. Tumor stromagenesis was repressed in Wnt $10 a^{-1-}$ mice. A microscopic examination showed that the pigmented melanomas in Wnt $10 a^{-1-}$ mice were significantly associated with more repressed proliferation of $\alpha$-SMA- or CD31-positive microvessels than in WT mice ( $\mathrm{n}=8$ mice per group), although only in cases where WNT10A and $\beta$-catenin co-expression was apparent, especially in tumor vessels. Accordingly, there were significantly fewer microvessels in Wnt l Oa ${ }^{-1-}$ mice than in WT mice. Scale bars $=100 \mu \mathrm{m}$. Values are means \pm SE. $* \mathrm{P}<0.05$, ***P $<0.0001$. 
A

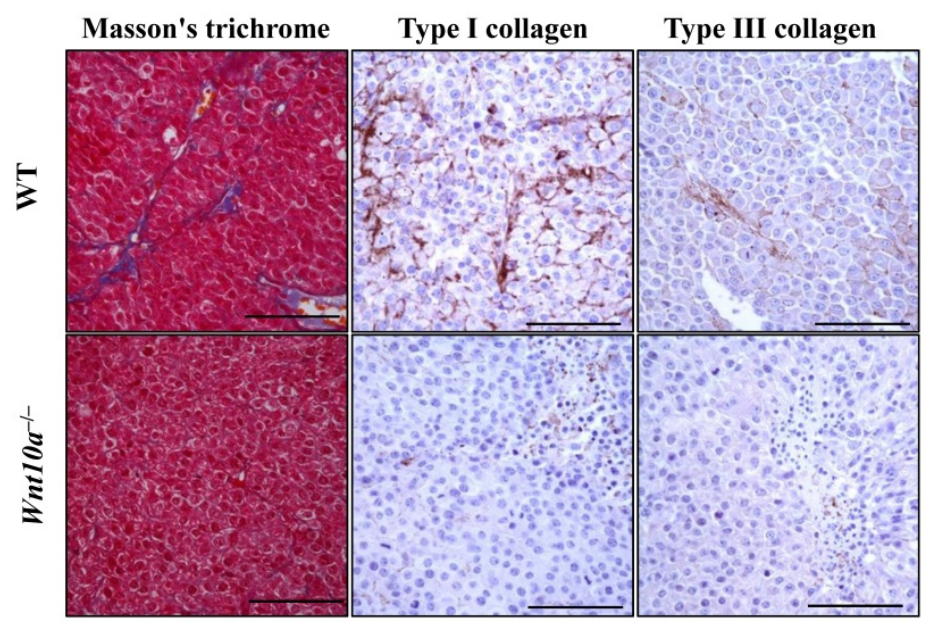

B

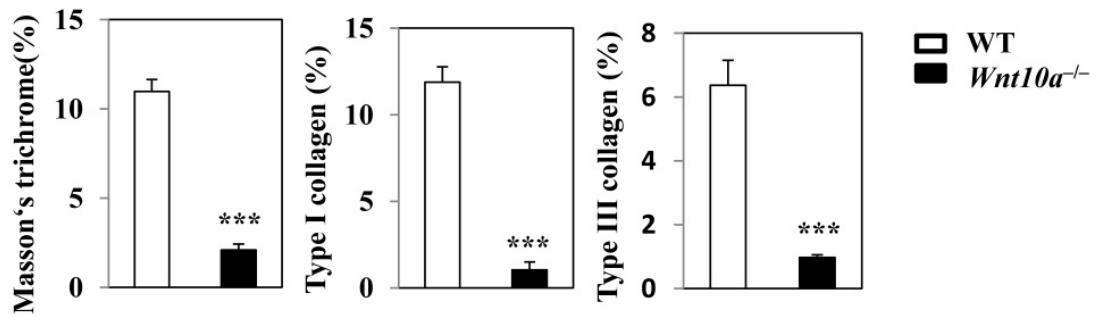

Figure 4. Reduced stromagenesis was closely associated with collagen expression. Masson's trichrome staining shows that there are significantly smaller amounts of blue-stained collagenous stroma (i.e. more suppressed fibrosis/fibrogenesis) in the transplanted tumor lesions of Wnt lOa-1- mice than in those of WT mice. Accordingly, IHC demonstrated that Wnt $\mathrm{Oa}^{-1-}$ transplanted melanomas had significantly lower expression of Type I/III collagen than WT transplanted melanomas, findings that were obvious in a large number of tumor vessel walls. Scale bars $=100 \mu \mathrm{m}$. Values are means $\pm \mathrm{SE}$. $* \mathrm{P}<0.05$, ***P $<0.0001$.

\section{Discussion}

In this study, Wnt10a-/- mice showed a smaller tumor size in the melanoma transplantation model than WT mice. In addition, tumor stromagenesis, including fibroblasts/myofibroblasts and angiogenesis/microvessels, was decreased in the melanoma tissues of Wnt10a-/- mice, leading to a larger necrosis area in the tumor tissues of these mice. Furthermore, in accordance with stromagenesis, reduced collagen expression was also observed in the Wnt10a-/-tumor tissues. The present results support the notion that WNT10A signaling may play a crucial role in the progression of malignant tumors (Figure 5).

Our previous results showed that WNT10A overexpression significantly promoted the proliferation of microvascular endothelial cells and fibroblasts/myofibroblasts in nude mouse xenograft models with injection of HeLa cells [16]. In line with these data, Wnt10a-/- mice in a murine melanoma B16-F10 cell transplantation model intriguingly displayed significantly repressed tumor growth, which was associated with more suppressed stromagenesis due to the presence of fewer microvessels and less fibrosis and more necrosis than in WT mice. Although Wnt10a-/- mice in the current study appear to have some differences in phenotype, compared to Wnt10a null mice generated by other labs, probably due to some differences in gene deletion sites [26], the deficiency of WNT10A in mice might demonstrate an anti-tumorigenic phenotype in a background of stromagenic failure.

These seeming discrepancies are considered to be based on the old hypothesis of cancer as an unhealing, overhealing 'wound' [13-14]. Indeed, recently, we reported that Wnt10a signaling was involved in the process of wound healing as a fibrogenic factor by regulating collagen expression [12]. Although the remarkable cellular parallels between skin wounds and malignant tumors have been followed with interest worldwide, tumors are completely different from conventional healing 'wounds', with no relevance to the "wounds that do not heal" or "over-healing wounds" hypotheses postulated by Dvorak and Haddow, respectively [27-28]. In fact, the tumor development process is not self-limited by uncontrolled proliferation and subsequent invasion and metastasis, in which cancer-associated fibroblasts (CAFs) are key, central players as hallmarks of cancer growth correlated with 


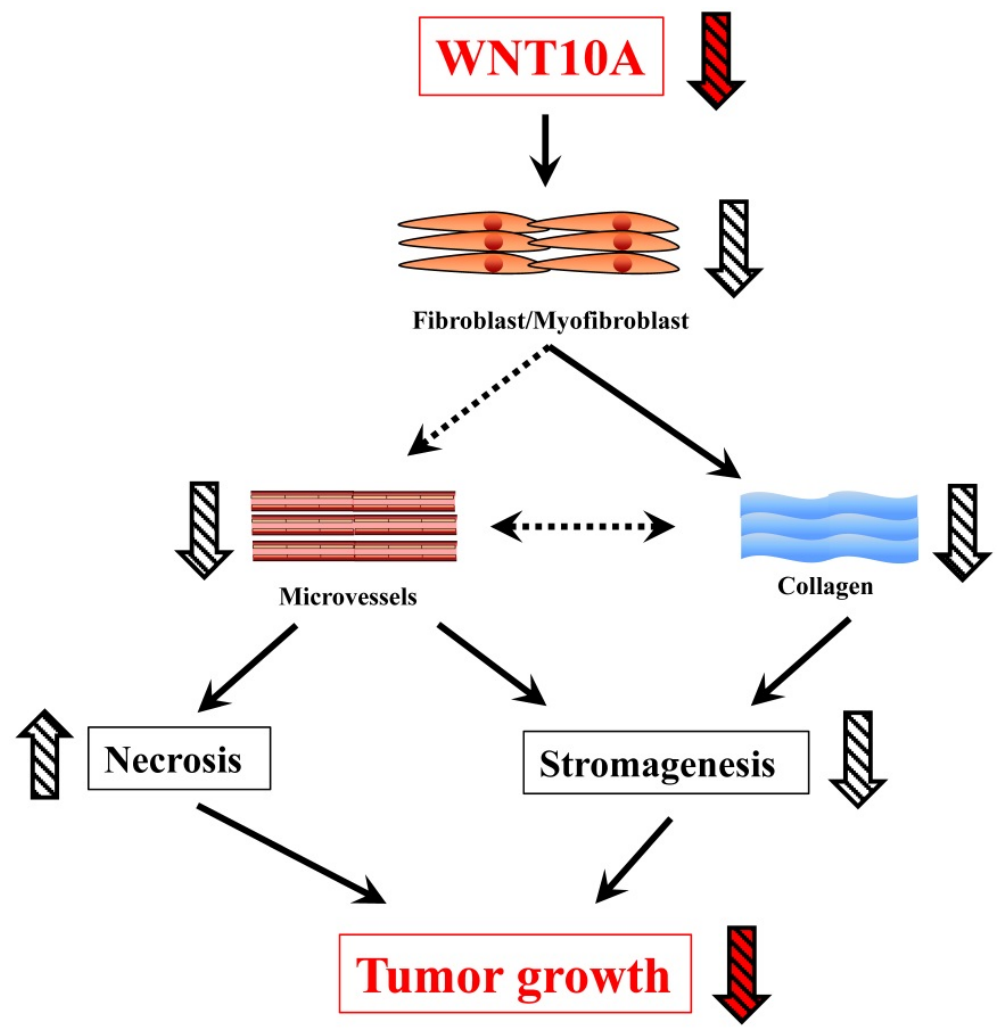

Figure 5. A schematic presentation of the critical in vivo roles of WNT10A in skin tumor.

fibrogenesis and ECM (collagen) expression/ synthesis [29]. Intriguingly, the action of CAFs can be stimulated by the disruption of circadian rhythms, which can promote cancer initiation and progression via WNT10A-dependent angiogenesis and fibrogenesis, accompanied by increased levels of oxidative stressors [16, 29].

Of note, the expression of $\beta$-catenin was significantly lower in the tumor tissues of Wnt10a-/mice than in those of WT mice in the murine melanoma B16-F10 cell transplantation model. The canonical WNT10A signaling pathway during stromagenesis is reportedly most likely stimulated by various immune system mediators, such as tumor necrosis factor (TNF)- $\alpha$ or nuclear factor (NF)-кB [6, 29-30], and/or various oxidative stressors [16, 31]. Moreover, due to the fact that mice blood cells expressing low level of Wnt10a (data not shown), its immune functions may be regulated by WNT10A signal in the initiation and progression of malignancy. Furthermore, the increased expression of $\beta$-catenin is regulated by the activation of upstream WNT10A signaling [2, 5]. Thus, in the present study, the deficiency of WNT10A may directly result in the reduced expression of $\beta$-catenin in $W n t 10 a^{-/-}$mice.

The exact role of $\mathrm{Wnt} / \mathrm{B}$-catenin signaling in melanoma remains controversial. Some researchers reported that Wnt-3a/beta-catenin signaling activity reduces B16-F1 melanoma tumor growth and metastasis by increasing differentiation [32], and Wnt10b can also inhibit proliferation and promote differentiation of B16-F10 melanoma cells [33], but many previous other studies showed that activation of Wnt/ß-catenin signaling can promote proliferation in melanoma cells [34-35]. These results may imply that different member in WNT glycoproteins family may play different roles in malignancy progression. WNT10A may be very different from other members. No strong expression of WNT10A was observed in tumor cells (Figure 3). Thus, we did not focus on tumor cells themselves, but gave more attentions to the role of Wnt10a/ß-catenin in tumor stromagenesis, including fibroblasts/myofibroblasts and angiogenesis/microvessels, which can promote tumor growth. In the present study, we therefore specially observed effect of WNT10A deficiency on the growth of melanoma cells in tumor microenvironment. However, due to the importance of Wnt/ß-catenin signal in proliferation and differentiation of tumor cells, the role of Wnt10a/ß-catenin signaling in melanoma cells need to be investigated in future study.

According to a growing number of experimental studies from our group, Y-box binding protein-1 (YB-1), a member of the cold shock protein family and regulator of cyclin expression, is highly expressed in tumor cells and tumor-associated stromal cells, including endothelial cells, making it a strong predictor for cancer aggressiveness and a poor prognosis, e.g. glioblastoma or advanced gastric cancer associated with vascular invasion and liver metastases [36-38]. However, YB-1 is also significantly regulated by day-night rhythms [39], potentially serving as a bridge between the circadian clock and cancer cell cycle control, similar to WNT10A. Further detailed studies are necessary to examine the intriguing relationships between WNT10A and YB-1, as well.

In conclusion, our present results showed that the deficiency of WNT10A significantly inhibited the growth of the tumor by reducing stromagenesis and promoting necrosis. These findings imply that its blockers may be promising therapeutic modalities against the initiation of the progression of malignant tumors by suppressing various aspects of stromagenesis, including fibroblasts/myofibroblasts and microvessels. Gene therapy with RNA interference (RNAi) for WNT10A may also be feasible. 


\section{Abbreviations}

Wnt10a-/-: Wnt10a-deficient mice; WT: wild-type; ECM: extracellular matrix; a-SMA: a-smooth muscle actin; ESCs: embryonic stem cells; RT-PCR: reverse transcription polymerase chain reaction; DMEM: Dulbecco's modified Eagle's minimal essential medium; FBS: fetal bovine serum; IHC: immunohistochemistry; H\&E: hematoxylin and eosin; CAFs: cancer-associated fibroblasts; TNF: tumor necrosis factor; YB-1: Y-box binding protein-1.

\section{Acknowledgments}

We would like to thank Ryoko Maekado (Shared-Use Research Center, School of Medicine, University of Occupational and Environmental Health) for her expert technical assistance. This work was supported in part by Grants-in-Aid for Scientific Research (JSPS KAKENHI) (Grant Numbers: JP15K09541 to Hiroto Izumi, 22249025 to Kimitoshi Kohno, \& 20590416 and 24790394 to Yasuyuki Sasaguri and Sohsuke Yamada) from the Ministry of Education, Culture, Sports, Science and Technology, Tokyo, Japan; a grant from the MSD Life Science Foundation, Public Interest Incorporated Foundation, Japan (to S.Y.); a UOEH grant for advanced research (to Ke-Yong Wang); grants from National Natural Science Foundation of China (No. 81402490) (to X.G.), Natural Science Foundation of Hebei Province (No. H2016206170) (to X.G.), and High level talent support project of Hebei Province (No. CG2015003011) (to X.G.), a grant for Promoted Research from Kanazawa Medical University (S2018-6) (to X.G.).

\section{Competing Interests}

The authors have declared that no competing interest exists.

\section{References}

1. Nusse R. Wnt signaling in disease and in development. Cell Res. 2005; 15: 28-32.

2. Kohn AD, Moon RT. Wnt and calcium signaling: beta-cateninindependent pathways. Cell Calcium. 2005; 38: 439-446.

3. Carroll TJ, Das A. Planar cell polarity in kidney development and disease. Organogenesis. 2011; 7: 180-190.

4. Lan Y, Jia S, Jiang R. Molecular patterning of the mammalian dentition. Semin Cell Dev Biol. 2014; 25-26: 61-70.

5. Liu F, Millar SE. Wnt/beta-catenin signaling in oral tissue development and disease. J Dent Res. 2010; 89: 318-330.

6. Klaus A, Birchmeier W. Wnt signalling and its impact on development and cancer. Nat Rev Cancer. 2008; 8: 387-398.

7. Kikuchi A, Yamamoto H, Kishida S. Multiplicity of the interactions of Wnt proteins and their receptors. Cell Signal. 2007; 19: 659-671.

8. Adaimy L, Chouery E, Megarbane H, Mroueh S, Delague V, Nicolas E, Belguith $\mathrm{H}$, de Mazancourt P, Megarbane A. Mutation in WNT10A is associated with an autosomal recessive ectodermal dysplasia: the odonto-onycho-dermal dysplasia. Am J Hum Genet. 2007; 81: 821-828.

9. Bohring A, Stamm T, Spaich C, Haase C, Spree K, Hehr U, Hoffmann M, Ledig S, Sel S, Wieacker P, Röpke A. WNT10A mutations are a frequent cause of a broad spectrum of ectodermal dysplasias with sex-biased manifestation pattern in heterozygotes. Am J Hum Genet. 2009; 85: 97-105.

10. Nawaz S, Klar J, Wajid M, Aslam M, Tariq M, Schuster J, Baig SM, Dahl N. WNT10A missense mutation associated with a complete odonto-onycho-dermal dysplasia syndrome. Eur J Hum Genet. 2009; 17: $1600-1605$.

11. Cluzeau C, Hadj-Rabia S, Jambou M, Mansour S, Guigue P, Masmoudi S, Bal E, Chassaing N, Vincent MC, Viot G, Clauss F, Manière MC, Toupenay S, Le Merrer M, Lyonnet S, Cormier-Daire V, Amiel J, Faivre L, de Prost Y, Munnich A, Bonnefont JP, Bodemer C, Smahi A. Only four genes (EDA1, EDAR, EDARADD, and WNT10A) account for $90 \%$ of hypohidrotic/anhidrotic ectodermal dysplasia cases. Hum Mutat. 2011; 32: 70-72.

12. Wang KY, Yamada S, Izumi H, Tsukamoto M, Nakashima T, Tasaki T, Guo X, Uramoto H, Sasaguri Y, Kohno K. Critical in vivo roles of WNT10A in wound healing by regulating collagen expression/synthesis in WNT10A-deficient mice. PLoS One. 2018; 13: e0195156.

13. Schäfer M, Werner S. Cancer as an overhealing wound: an old hypothesis revisited. Nat Rev Mol Cell Biol. 2008; 9: 628-638.

14. Dvorak HF. Tumors: wounds that do not heal. Similarities between tumor stroma generation and wound healing. N Engl J Med. 1986; 315: 1650-1659.

15. Darby IA, Laverdet B, Bonté F, Desmoulière A. Fibroblasts and myofibroblasts in wound healing. Clin Cosmet Investig Dermatol. 2014; 7: 301-311.

16. Yasuniwa Y, Izumi H, Wang KY, Shimajiri S, Sasaguri Y, Kawai K, Kasai H, Shimada T, Miyake K, Kashiwagi E, Hirano G, Kidani A, Akiyama M, Han B, $\mathrm{Wu}$ Y, Ieiri I, Higuchi S, Kohno K. Circadian disruption accelerates tumor growth and angio/stromagenesis through a Wnt signaling pathway. PLoS One. 2010; 5: e15330.

17. Wang KY, Tanimoto A, Yamada S, Guo X, Ding $Y$, Watanabe $T$, Watanabe $T$, Kohno K, Hirano K, Tsukada H, Sasaguri Y: Histamine regulation in glucose and lipid metabolism via histamine receptors: model for nonalcoholic steatohepatitis in mice. Am J Pathol. 2010; 177: 713-723.

18. Wang KY, Tanimoto A, Guo X, Yamada S, Shimajiri S, Murata Y, Ding $Y$, Tsutsui M, Kato S, Watanabe T, Ohtsu H, Hirano K, Kohno K, Sasaguri Y: Histamine deficiency decreases atherosclerosis and inflammatory response in apolipoprotein E knockout mice independently of serum cholesterol level. Arterioscler Thromb Vasc Biol. 2011; 31: 800-807.

19. Yamada S, Ding Y, Tanimoto A, Wang KY, Guo X, Li Z, Tasaki T, Nabesima A, Murata Y, Shimajiri S, Kohno K, Ichijo H, Sasaguri Y: Apoptosis signal-regulating kinase 1 deficiency accelerates hyperlipidemia-induced atheromatous plaques via suppression of macrophage apoptosis. Arterioscler Thromb Vasc Biol. 2011; 31: 1555-1564.

20. Seong I, Min HJ, Lee JH, Yeo CY, Kang DM, Oh ES, et al. Sox10 controls migration of B16F10 melanoma cells through multiple regulatory target genes. PLoS One. 2012; 7: e31477.

21. Khosravi-Maharlooei M, Pakyari M, Jalili RB, Salimi-Elizei S, Lai JC, Poormasjedi-Meibod $\mathrm{M}$, et al. Tolerogenic effect of mouse fibroblasts on dendritic cells. Immunology. 2016; 148: 22-33.

22. Kranjc S, Kranjc M, Scancar J, Jelenc J, Sersa G, Miklavcic D. Electrochemotherapy by pulsed electromagnetic field treatment (PEMF) in mouse melanoma B16F10 in vivo. Radiol Oncol. 2016; 50: 39-48.

23. Yamada S, Wang KY, Tanimoto A, Fan J, Shimajiri S, Kitajima S, et al. Matrix metalloproteinase 12 accelerates the initiation of atherosclerosis and stimulates the progression of fatty streaks to fibrous plaques in transgenic rabbits. Am J Pathol. 2008; 172: 1419-1429.

24. Tasaki T, Yamada S, Guo X, Tanimoto A, Wang KY, Nabeshima A, et al. Apoptosis signal-regulating kinase 1 deficiency attenuates vascular injury-induced neointimal hyperplasia by suppressing apoptosis in smooth muscle cells. Am J Pathol. 2013; 182: 597-609.

25. Noguchi H, Yamada S, Nabeshima A, Guo X, Tanimoto A, Wang KY, et al. Depletion of apoptosis signal-regulating kinase 1 prevents bile duct ligation-induced necroinflammation and subsequent peribiliary fibrosis. Am J Pathol. 2014; 184: 644-61.

26. Xu M, Horrell J, Snitow M, Cui J, Gochnauer H, Syrett CM, Kallish S, Seykora JT, Liu F, Gaillard D, Katz JP, Kaestner KH, Levin B, Mansfield C, Douglas JE, Cowart BJ, Tordoff M, Liu F, Zhu X, Barlow LA, Rubin AI, McGrath JA, Morrisey EE, Chu EY, Millar SE. WNT10A mutation causes ectodermal dysplasia by impairing progenitor cell proliferation and KLF4-mediated differentiation. Nat Commun. 2017; 8: 15397.

27. Haddow A. Molecular repair, wound healing, and carcinogenesis: tumor production a possible overhealing? Adv Cancer Res. 1972; 16: 181-234.

28. Dvorak HF. Tumors wounds that do not heal. Similarities between tumor stroma generation and wound healing. N Engl J Med. 1986; 315: 1650-1659.

29. Izumi H, Wang K, Morimoto Y, Sasaguri Y, Kohno K. Circadian disruption and cancer risk: a new concept of stromal niche (review). Int J Oncol. 2014; 44: $364-370$.

30. Kirikoshi H, Sekihara H, Katoh M. Up-regulation of WNT10A by tumor necrosis factor alpha and Helicobacter pylori in gastric cancer. Int J Oncol. 2001; 19: 533-536.

31. Kuma A, Yamada S, Wang KY, Kitamura N, Yamaguchi T, Iwai Y, et al. Role of WNT10A-expressing kidney fibroblasts in acute interstitial nephritis. PLoS One. 2014; 9: e103240.

32. Chien AJ, Moore EC, Lonsdorf AS, Kulikauskas RM, Rothberg BG, Berger AJ, Major MB, Hwang ST, Rimm DL, Moon RT. Activated Wnt/beta-catenin signaling in melanoma is associated with decreased proliferation in patient tumors and a murine melanoma model. Proc Natl Acad Sci U S A. 2009; 106: 1193-8.

33. Misu M, Ouji Y, Kawai N, Nishimura F, Nakamura-Uchiyama F, Yoshikawa M. Effects of Wnt-10b on proliferation and differentiation of murine melanoma cells. Biochem Biophys Res Commun. 2015; 463: 618-23. 
34. Moon RT, Kohn AD, De Ferrari GV, Kaykas A. WNT and beta-catenin signalling: diseases and therapies. Nat Rev Genet. 2004; 5: 691-701.

35. WidlundHR, Horstmann MA, Price ER, Cui J, Lessnick SL, Wu M, He X, Fisher DE. Beta-catenin-induced melanoma growth requires the downstream target Microphthalmia-associated transcription factor. J Cell Biol. 2002; 158: 1079-87.

36. Takahashi M, Shimajiri S, Izumi H, Hirano G, Kashiwagi E, Yasuniwa Y, et al. Y-box binding protein-1 is a novel molecular target for tumor vessels. Cancer Sci. 2010; 101: 1367-1373.

37. Wu Y, Yamada S, Izumi H, Li Z, Shimajiri S, Wang KY, et al. Strong YB-1 expression is associated with liver metastasis progression and predicts shorter disease-free survival in advanced gastric cancer. J Surg Oncol. 2012; 105: $724-730$.

38. Wu Y, Wang KY, Li Z, Liu YP, Izumi H, Yamada S, et al. Y-box binding protein 1 expression in gastric cancer subtypes and association with cancer neovasculature. Clin Transl Oncol. 2015; 17: 152-159.

39. Pagano C, di Martino O, Ruggiero G, Maria Guarino A, Mueller N, Siauciunaite $\mathrm{R}$, et al. The tumor-associated YB-1 protein: new player in the circadian control of cell proliferation. Oncotarget. 2017; 8: 6193-6205. 